\title{
Equação de chuvas para Fortaleza-CE com dados do pluviógrafo da UFC
}

\author{
Rainfall equation for Fortaleza based on pluviograph data from UFC
}

\author{
Francisco Osny Enéas da Silva | Francisco Flávio Rocha Palácio Júnior \\ José Nilson Bezerra Campos
}

Data de entrada: 05/04/2011 | Data de aprovação: 18/12/2012

Resumo

O artigo apresenta uma equação de chuvas para a cidade de Fortaleza a partir de 30 anos de registros pluviográficos (1970-1999) da estação climatológica da Universidade Federal do Ceará. Foram avaliadas séries anuais de eventos de intensidades médias máximas de chuvas para durações de 5, 10, 20, 30, 45, 60 e 120 minutos. O processo resultou da análise de 611 hietogramas de precipitação observados no período. Na determinação dos parâmetros da equação Intensidade-Duração-Frequência (IDF), aplicou-se o método de Gumbel-Chow. Os dados foram ajustados a uma distribuição de probabilidade Gumbel e para definição da equação de chuva aplicou-se o método descrito por Wilken (1978). A equação permite calcular diretamente as intensidades de precipitação para períodos de retorno de 5 a 100 anos para durações de chuva de até 120 minutos. Os resultados da nova equação foram comparados com os valores obtidos pela aplicação de três equações anteriores, sendo que as duas primeiras foram desenvolvidas nas décadas de 70 e 80 do século XX e, a terceira, no ano de 2008.

Palavras-chave: Equação de chuva, registros pluviográficos, Curvas intensidade-duração-frequência.

\section{Abstract}

The article introduces an intensity-duration-frequency (IDF) equation for the city of Fortaleza from 30 years of pluviographic records (1970-1999) of the climatological station of the Federal University of Ceará. The equation was built based on annual series of maximum averages of rainfall intensities for durations of 5, 10,20, 30, 45, 60 and 120 minutes. The case resulted from the analysis of 611 rainfall hyetograph observed in the period. In determining the parameters of the equation IDF was applied the Gumbel-Chow method. The data were adjusted to a Gumbel probability distribution function, and to defining the rain equation applied the method described by Wilken (1978). The equation is valid for return periods from 5 to 100 years and for rainfall durations up to 120 minutes. The results of the new equation were compared with values obtained by the application of three previous equations. The first two were developed in the 70's and 80's of the twentieth century, and the third, in year 2008.

Key-words: IDF equation, pluviograph data, Intensity-duration-frequency curves.

Francisco Osny Enéas da Silva*

Engenheiro Civil, Doutor em Recursos Hídricos, Universidade Federal do Ceará, Professor Adjunto da Universidade Estadual Vale do Acaraú - UVA

Francisco Flávio Rocha Palácio Júnior

Engenheiro Civil, Departamento Estadual de Pavimentação e Saneamento DEPASA - Rio Branco, Acre

José Nilson Bezerra Campos

Engenheiro Civil, PhD em Recursos Hídricos, Colorado State University, Professor Titular da Universidade Federal do Ceará - UFC

\footnotetext{
* Endereço para correspondência: (pessoal) Av. Cel Carvalho 3078, Fortaleza-Ce, CEP 60351-364, Tel: +55 (85) 3286-1823; (profissional) Universidade Vale do Acaraú, Coordenação de Engenharia Civil, Campus CIDAO, Av. Dr. Guarany 317, Derby Club, Sobral-Ce, CEP 62040-730, Tel: +55 (88) 3611-6547. E-mail: osnyeneassilva@gmail.com
} 


\section{Introdução}

Os modelos comumente empregados na transformação de chuva em vazão em projetos de Drenagem Urbana (DU) são os modelos ditos concentrados, tais como a fórmula racional, os métodos da onda cinemática e os métodos relacionados ao hidrograma unitário. Com uma menor aplicação pelos projetistas de drenagem urbana aparecem os modelos de abordagem distribuída e semi-distribuída como o modelo Topmodel (BEVEN et. al., 1995, XAVIER, 2002). A determinação da vazão de projeto para dimensionamento de uma dada estrutura hidráulica é feita em duas etapas: inicialmente, determina-se a chuva de projeto representada por um hietograma; em seguida, aplica-se o hietograma em um modelo chuva deflúvio.

A escolha do hietograma e do modelo chuva $\mathrm{x}$ vazão depende basicamente da escala da bacia hidrográfica. Trata-se, em síntese, de selecionar as distribuições espaciais e temporais da chuva de projeto. Para bacias pequenas, menores do que $2,5 \mathrm{~km}^{2}$ admite-se a chuva com intensidade constante em toda sua duração e distribuição uniforme ao longo da bacia hidrográfica. Para bacias médias, menores do que $100 \mathrm{~km}^{2}$ admite-se que a chuva varia ao longo do tempo e é uniforme no espaço. Para grandes bacias, aplicam-se modelos com chuvas variáveis no tempo e no espaço (PONCE, 1989).

Nas bacias urbanas, em geral, adotam-se as premissas de bacias pequenas e médias. Em ambos os casos, quer seja por precipitação constante ou variável no tempo, a equação intensidade- duração-frequência (IDF) é de fundamental importância para a formulação da chuva de projeto.

Os dimensionamentos das obras de microdrenagem urbanas, tais como as sarjetas, as bocas-de-lobo, as galerias de drenagem pluvial e, os bueiros, são feitos com base no emprego de equações intensidade-duração-frequência, conhecidas no meio técnico de engenharia como equações de chuva.

O DNIT, órgão nacional gestor do sistema rodoviário, exige a elaboração de curvas de intensidade-duração-frequência (I-D-F) e de altura-duração-frequência (A-D-F), para períodos de retorno de $5,10,15,25,50$ e 100 anos, como pressuposto para o dimensionamento de obras rodoviárias, tais como os bueiros e os pontilhões (DNIT, 2006). A síntese destas curvas conduz à elaboração de equações de chuva.
Atualmente, em Fortaleza, a Secretaria de Infraestrutura Municipal (SEINF) ainda utiliza nos projetos de Drenagem Urbana (DU) a primeira equação de chuva desenvolvida em 1978 no âmbito do Plano Diretor de Drenagem Urbana de Fortaleza (CEARÁ, 1978). Há uma segunda equação de chuva desenvolvida em 1983 pelos professores Clóvis Eduardo Matos Neto e Nise Sanford Fraga, em uma pesquisa na Universidade Federal do Ceará (MATOS NETO e FRAGA, 1983) e, uma terceira equação desenvolvida por Rodrigues et.al (RODRIGUES et. al., 2008).

Zuffo (2004) discute sobre a questão da necessidade de revisão periódica das equações de chuva orientadoras de projeto de drenagem urbana pelo menos uma vez em cada década, apresentado na sua publicação "Equações de Chuva São Eternas?" no XXI Congresso Latino Americano de Hidráulica realizado em São Paulo em 2004. Nessa lógica foi desenvolvida uma nova equação de chuvas para Fortaleza com dados da estação pluviográfica da Universidade Federal do Ceará. A oportunidade decorreu da obtenção de dados digitalizados a partir dos registros pluviográficos organizados pelo Engenheiro Vicente Melo Lima no âmbito de sua dissertação de mestrado para o período de 1970 a 1999 (LIMA, 2005).

O objetivo do presente trabalho é apresentar uma nova equação IDF para Fortaleza com base nos dados da Estação Climatológica da Universidade Federal do Ceará, localizada no Campus do Pici na parte Oeste da cidade, e discutir a variação dos resultados obtidos pela comparação do emprego da nova equação com as três equações anteriores.

\section{A área de estudo}

A cidade de Fortaleza, capital do Estado do Ceará, com uma população de 2.447.409 habitantes (BRASIL, 2011) é a quinta cidade mais populosa do país e a segunda maior em densidade demográfica. A Região Metropolitana de Fortaleza (RMF) engloba 15 municípios e tem uma população de aproximadamente $3,6 \mathrm{mi}$ lhões de habitantes, sendo a sexta maior cidade do país e se inclui entre as 80 maiores regiões metropolitanas do mundo (CEARÁ, 2011, CITYMAYORS, 2010). A Figura 1 mostra o mapa da região metropolitana de Fortaleza localizada no Estado do Ceará. 


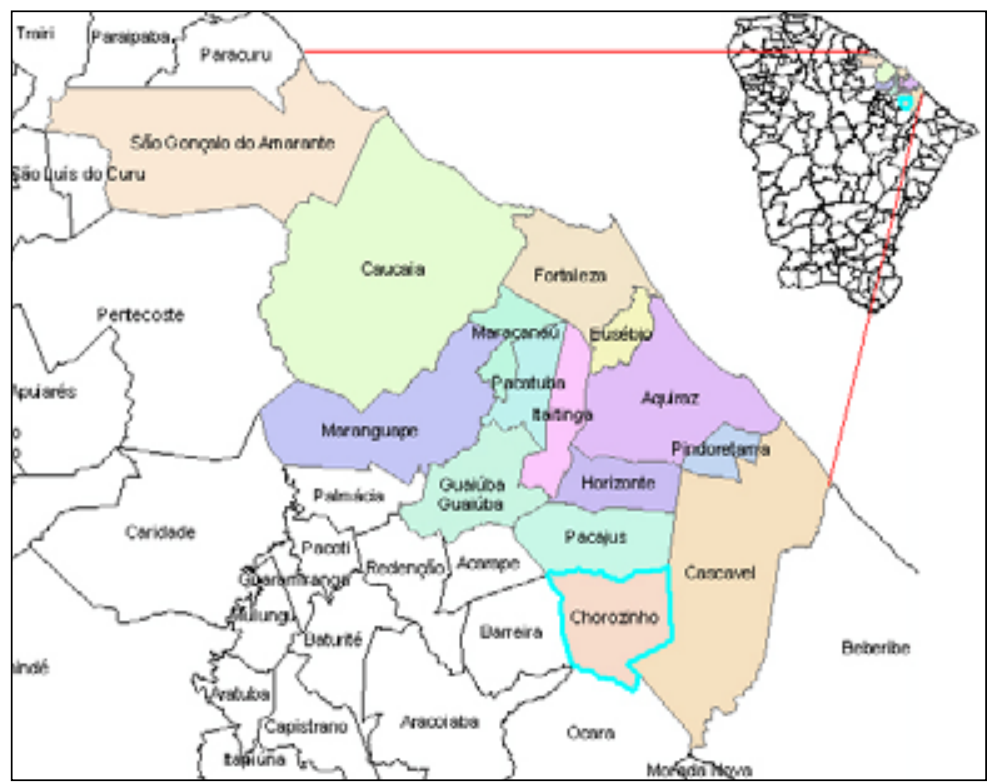

Fonte: Banco de dados dos autores, 2011

Figura 1- Mapa da Região Metropolitana de Fortaleza no Estado do Ceará.

\section{Base conceitual}

De forma geral, as relações entre intensidade, duração e frequência das precipitações pluviais podem ser representadas por equações do tipo mostrado na Equação 1 (MARTINEZ JR. e MAGNI, 1999):

Os valores de $\mathrm{C}$ e $\mathrm{n}$ variam com o período de

$$
i=\frac{C}{\left(t+t_{0}\right)^{n}}
$$

\section{Onde}

$i$ = intensidade pluviométrica média máxima para a duração

\section{t, em $\mathrm{mm} / \mathrm{min}$}

$t=$ duração da chuva em minutos

$C$, ne $t_{0}$ : são os parâmetros empíricos.

retorno $\mathrm{T}$.

As relações entre $\mathrm{C}$ e $\mathrm{T}$ e $\mathrm{n}$ e $\mathrm{T}$ podem ser representadas vários tipos de equações (MAGNI e MELO, 1986).

Em uma delas, admite-se n como constante e introduz-se um novo parâmetro na equação, na forma da Equação 2:

A inserção da Equação 2 na Equação 1 resulta

$$
C=K T^{m}
$$

Onde

K e m são parâmetros da equação

$T$ é o período de retorno em anos

na Equação 3:

Vários autores reportam a forma da Equação 3

$$
i=\frac{K \cdot T^{m}}{\left(t+t_{0}\right)^{n}}
$$

Onde

i representa a intensidade da precipitação $(\mathrm{mm} / \mathrm{h})$

T denota o tempo de retorno (anos)

$t$ a duração da chuva (minutos)

$K, m, t_{0}$, n são parâmetros da equação obtidos

empiricamente para cada localidade 
como a mais utilizada em representar as relações IDF de chuvas intensas (CAMPOS, 2009, VILELLA e MATTOS, 1975, PONCE, 1989).

Para obtenção dos parâmetros da Equação IDF, os dados podem ser organizados em séries anuais ou em séries parciais. As séries anuais são formadas pelo valor máximo da precipitação observada em cada ano para uma determinada duração. As séries parciais são formadas pelos maiores valores de precipitações independentes, de cada duração, acima de um dado valor de referência, sem levar em conta o número de ocorrências dentro de um dado período.

Muitos engenheiros e pesquisadores já desenvolveram equações IDF. Entre estes podem ser citados: Pinto et al. (1996) no Estado de Minas Gerais, Fendrich (1998) no Estado do Paraná, Costa \& Brito (1999) e Oliveira et al. (2000) no Estado de Goiás, Martinez Júnior (1999) no Estado de São Paulo, Silva et al. (1999) para o Estado do Rio de Janeiro e Espírito Santo, Back (2009) e Nerilo et al. (2002) para o Estado de Santa Catarina.

Devido à dificuldade na obtenção dos dados pluviográficos, a grande maioria dos estudos de chuvas intensas possui series inferiores àquela recomendada pela Organização Mundial de Meteorologia (OMM), que é de 30 anos.

Pfafstetter (1982) no ano de 1957 desenvolveu um trabalho pioneiro denominado Chuvas Intensas no Brasil, que utilizou séries de valores máximos de precipitações de 98 estações pluviométricas em diversas regiões do país, para a construção de curvas intensidade-duração-frequência, utilizando a distribuição de Gumbel. O procedimento utilizado para a seleção dos maiores eventos de chuvas neste trabalho é considerado um clássico dentro da área. Para uma primeira seleção de eventos de chuvas intensas, adotou-se para as durações de 5, 15 e 30 minutos, e 1, 2, 4, $8,14,24$ e 48 horas e os limites de precipitação mínimos de 8, 15, 20, 25, 30, 35, 40, 47, 55 e 70 milímetros respectivamente, segundo critérios propostos por Pfafstetter. O autor selecionou, em média, aproximadamente 10 chuvas em cada ano de observação, para cada posto pluviométrico, porque, em geral, as chuvas intensas não apresentam precipitações que excedem os limites mínimos fixados para todas as durações.

O trabalho de Pfafstetter (1982) foi incorporando no conjunto das equações de chuva que estão disponíveis no aplicativo ABC6, desenvolvido no Laboratório de Sistemas de Suporte à Decisão da Escola Politécnica da Universidade de São Paulo (OLIVEIRA et. al., 1999).

\section{A equação da AUMEF}

Em 1976, a Autarquia Metropolitana de Fortaleza (AUMEF) contratou com a Empresa de Consultoria Aqua Plan a elaboração de um Plano Diretor de Drenagem para a Região Metropolitana de Fortaleza. Na época, Fortaleza ainda não dispunha de uma equação IDF para utilização no dimensionamento das obras hidráulicas. Todos os dimensionamentos eram feitos com o método do Engenheiro Otto Pfafstetter desenvolvido em 1957.

A Consultora desenvolveu a primeira equação para Fortaleza com base em dados da estação climatológica do Inmet no período de 1928 a 1975. No final da análise de consistência dos dados foi utilizada uma série de 37 anos. (CEARÁ, 1978, p. 165).

A empresa chegou à Equação 4, válida para durações menores do que duas horas.

A equação da AUMEF ainda é muito aplicada

$$
i=\frac{528,076 \cdot T^{0,148}}{(t+6)^{0,62}}
$$

Onde

i denota a intensidade de precipitação $(\mathrm{mm} / \mathrm{h})$

Trepresenta o tempo de retorno (anos)

$t$ a duração da chuva (min)

em projetos de drenagem urbana na Região Metropolitana de Fortaleza e cidades próximas, sendo inclusive reconhecida como equação oficial pela Secretaria de Infraestrutura do Município de Fortaleza.

\section{A equação de Matos Neto e Fraga}

Matos Neto \& Fraga (1983) desenvolveram uma equação de chuvas intensas para Fortaleza. $\mathrm{Na}$ sua elaboração foram utilizados dados de pluviógrafo da estação meteorológica do Centro de Ciências Agrárias da Universidade Federal do Ceará referentes ao período de 1964 a 1980. Os autores empregaram a metodologia descrita por Wilken (1978) adotando o método de Chow-Gumbel para estimativa da frequência das preci- 
pitações intensas, segundo as durações de 5, 10, 20, 30, 45, 60 e 120 minutos. Os parâmetros da equação foram determinados pelo método dos mínimos quadrados. A fórmula obtida é apresentada na Equação 5.

$$
i=\frac{506,99 \cdot T^{0,181}}{(t+8)^{0,61}}
$$

Onde

i denota a intensidade de precipitação $(\mathrm{mm} / \mathrm{h})$

$T$ representa o tempo de retorno (anos)

t a duração da chuva (min)

\section{A equação de Rodrigues, Andrade, Oliveira e Lo- bato}

Rodrigues et. al. (2008) desenvolveram equações de chuvas para as cidades de Fortaleza e Pentecoste no Estado do Ceará, empregando o modelo teórico da distribuição de probabilidades de Gumbel aos dados da estação meteorológica de Fortaleza e Pentecoste, para as durações de 5, 10, 15, 20, 30, 45, 60, 90, 120, 180, 240, 360, 720 e 1440 minutos. O modelo probabilístico ajustado foi testado pelo teste de aderência de Kolmogorov-Smirnov, evidenciando ajuste em nível de 20\% de significância.

Segundo os próprios autores, os valores estimados pela equação geral podem apresentar erros de até $40 \%$ para a localidade de Pentecoste, confirmando a necessidade de equações distintas para cada período de retorno. Os dados ajustados aproximaram-se dos valores obtidos pela equação de Gumbel, evidenciando que as equações desenvolvidas podem ser empregadas na estimativa das intensidades de chuva, com exceção da duração de 5 minutos na estação de Fortaleza, pois há uma tendência em subestimar os valores esperados. A equação para Fortaleza é apresentada na Equação 6.

$$
i=\frac{18,926 \cdot T^{0,1621}}{(t+12)^{0,76}}
$$

\section{Dados e metodologia}

A nova equação de chuva para Fortaleza foi obtida através de análise estatística dos dados do pluviógrafo da estação meteorológica do Centro de Ciências Agrárias da Universidade Federal do Ceará, o qual dispõe de registros contínuos com intervalo de 5 em 5 minutos desde 1970 até 1999. Os dados utilizados foram digitalizados pelo Engenheiro Vicente Melo Lima para o desenvolvimento de sua dissertação de mestrado na Universidade Federal do Ceará (LIMA, 2005)

A equação foi desenvolvida em três fases: 1) a fase preparatória de organização dos dados; 2) a fase de estudos estatísticos para avaliação das relações frequenciais; 3) fase de determinação dos parâmetros da IDF. Complementarmente foi feita uma comparação com os resultados das equações anteriores.

\section{Fase Preparatória}

No presente trabalho, os dados de precipitação foram organizados em durações com intervalos de 5, 10, 20, 30, 45, 60 e 120 minutos. Para cada intervalo de tempo foi formada uma série com as precipitações máximas anuais. Em seguida, dividindo-se a precipitação pelo respectivo intervalo de tempo, formaram-se as séries anuais de intensidades médias máximas anuais.

$\mathrm{Na}$ análise das chuvas diárias, criaram-se hietogramas para as chuvas de maior intensidade observadas em cada ano. O objetivo principal da elaboração dos hietogramas foi facilitar a leitura e escolhas das chuvas máximas anuais. Durante o processo foram elaborados 611 hietogramas. A precipitação máxima diária ocorrida no período em estudo (1970 a 1999) ocorreu em 25/03/1995 correspondendo a $171,20 \mathrm{~mm}$.

\section{Análise Estatística}

Inicialmente foram calculadas as estatísticas amostrais média e desvio padrão para as sete durações de chuva (Tabela 1).

Em seguida, foi feito um ajustamento à distribuição de Gumbel para determinar as relações entre e as intensidades médias máximas e os respectivos períodos de retorno. Os períodos de retorno selecionados foram de 5, 10, 15, 20, 25, 50 e 100 anos com a aplicação da Equação 7.

$\mathrm{O}$ fator de frequência $\mathrm{K}$ varia com o período de retorno e com o tipo de distribuição estatística. 


\begin{tabular}{c|c|c|c|c|c|c|c} 
Duração & $5 \mathrm{~min}$ & $\mathbf{1 0} \mathrm{min}$ & $20 \mathrm{~min}$ & $30 \mathrm{~min}$ & $\mathbf{4 5} \mathrm{min}$ & $60 \mathrm{~min}$ & $120 \mathrm{~min}$ \\
\hline Média & 108,18 & 88,35 & 66,77 & 61,06 & 50,63 & 43,42 & 23,79 \\
\hline Desvio-padrão & 43,54 & 21,76 & 20,85 & 22,09 & 18,08 & 16,65 & 11,42
\end{tabular}

Tabela 1- Estatística das intensidades médias máximas na estação climatológica da UFC no período de 1970 a $1999(\mathrm{~mm} / \mathrm{h})$

$$
X=\bar{X}+K \cdot S
$$

Onde

$X$ = intensidade média máxima com período de retorno $T$;

$\underline{X}$ = intensidade média máxima da amostra;

$K$ = fator de frequência da distribuição de Gumbel;

$s=$ desvio padrão da amostra.

Para a distribuição de Gumbel utiliza-se a Equação 8:

$$
K=\frac{-\sqrt{6}}{\pi}\{\delta+\ln [\ln (T)-\ln (T-1)]\}
$$

Onde

$T=$ período de retorno

$\delta=$ constante de Euler igual a 0,5772157

Com a aplicação das equações 6 e 7 foram calculadas as relações entre as intensidades médias máximas e os períodos de retorno (Tabela 2).

\begin{tabular}{c|c|c|c|c|c|c|c|c|c}
\multirow{2}{*}{$\begin{array}{c}\text { Duração } \\
\text { (min) }\end{array}$} & $\begin{array}{c}\text { Dados } \\
\text { Estatísticos }\end{array}$ & \multicolumn{7}{|c}{ Intensidade Máxima Provável ( mm/hora) } \\
\cline { 2 - 11 } & Média & Desvio-padrão & 5 anos & 10 anos & 15 anos & 20 anos & 25 anos & 50 anos & 100 anos \\
\hline 5 & 108,18 & 43,54 & 139,5 & 165,0 & 179,4 & 189,4 & 197,2 & 221,0 & 244,7 \\
\hline 10 & 88,35 & 21,76 & 104,0 & 116,8 & 123,9 & 129,0 & 132,8 & 144,8 & 156,6 \\
\hline 20 & 66,77 & 20,85 & 81,8 & 94,0 & 100,9 & 105,7 & 109,4 & 120,8 & 132,2 \\
\hline 30 & 61,06 & 22,09 & 76,9 & 89,9 & 97,2 & 102,3 & 106,2 & 118,3 & 130,3 \\
\hline 45 & 50,63 & 18,08 & 63,6 & 74,2 & 80,2 & 84,4 & 87,6 & 97,5 & 107,3 \\
\hline 60 & 43,42 & 16,65 & 55,4 & 65,2 & 70,6 & 74,5 & 77,5 & 86,6 & 95,6 \\
\hline 120 & 23,79 & 11,42 & 32,0 & 38,7 & 42,5 & 45,1 & 47,1 & 53,4 & 59,6 \\
\hline
\end{tabular}

Tabela 2- Relações entre as intensidades médias de chuvas e respectivos períodos de retorno, calculadas pelo método de Gumbel-Chow. 


\section{Estimativa dos parâmetros da equação IDF}

Na determinação da Equação IDF, utilizou-se o método clássico descrito por Wilken (1978, p. 50). A forma geral da equação de chuvas intensas, para um dado período de retorno pode ser representada no domínio logarítmico pela Equação 9 .

$$
\log _{i}=\log A+b \cdot \log (t+c)
$$

\section{Onde}

$i=$ intensidade $\mathrm{em} \mathrm{mm} \cdot \mathrm{min}-1$

$t=$ duração da chuva em minutos

$b=$ tangente do ângulo de inclinação da reta

$A$ = intercepto, ou a ordenada correspondente à duração de um minuto

$c=o$ termo utilizado devido à necessidade de uma correção referente à duração

\section{Obtenção do valor de c}

Ao se traçar a curva duração $\mathrm{x}$ intensidade de precipitação em um diagrama bilogarítmico, com pares de pontos (t, i), observou-se um trecho em curva mais acentuada, localizado na região correspondente às menores durações. Assim, existe a necessidade de se fazer correção, introduzindo a

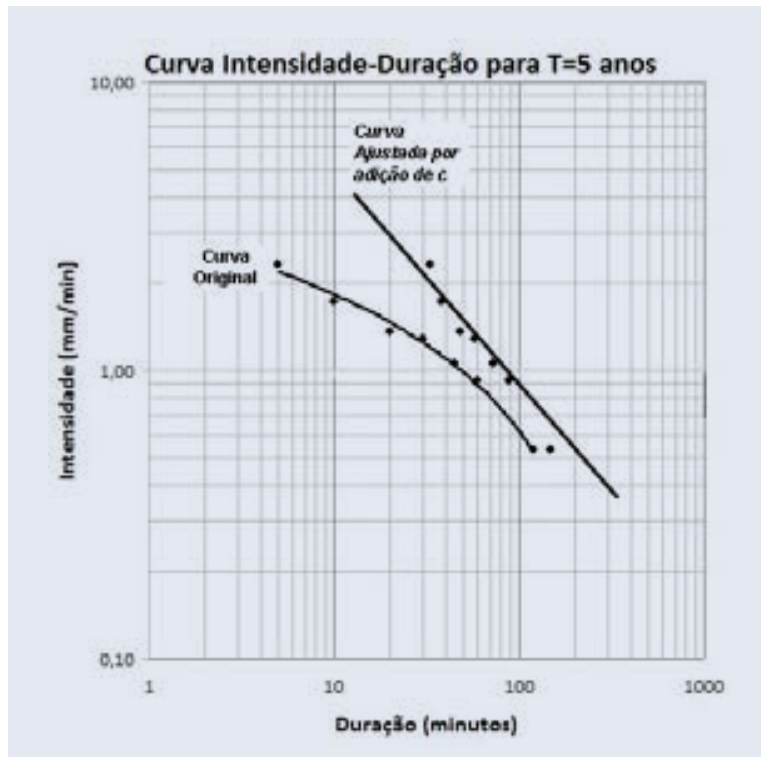

Figura 2 - Gráfico de intensidade-duração para período de retorno de cinco anos. constante c, no termo referente à duração de precipitação. Não há uma regra especifica para a determinação da constante c e sua determinação deve ser feita por tentativas. Segundo Wilken (1978), o valor de c deve ser estudado, empregando-se a curva intensidade-duração correspondente ao período de retorno de cinco anos. Esta curva é apresentada na Figura 2.

No gráfico bilogarítmico da Figura 2 para um tempo de retorno de cinco anos, inicialmente, é traçada uma curva provisória por interpolação visual (curva original). Em seguida, foram escolhidos nesta curva dois pontos próximos das extremidades, cujas coordenadas são respectivamente $\left(t_{1}, i_{1}\right)$ e $\left(t_{2}, i_{2}\right)$. A Equação 10 apresenta a relação matemática destes dois pontos em relação aos parâmetros da equação de chuva.

$$
i_{1}=\frac{A}{\left(t_{1}+c\right)^{b}} ; i_{2}=\frac{A}{\left(t_{2}+c\right)^{b}}
$$

Os pares de valores obtidos foram: $\left(t_{1}=7 \mathrm{~min}\right.$; $\left.i_{1}=2,0 \mathrm{~mm} / \mathrm{min}\right)$ e $\left(t_{2}=100 \mathrm{~min} ; i_{2}=0,6 \mathrm{~mm} / \mathrm{min}\right)$. Considerou-se, em seguida, um terceiro ponto $\left(t_{3}\right.$, $i_{3}$ ) na mesma curva. $\mathrm{O}$ valor de $i_{3}$ é obtido pela Equação 11 e $t_{3}$ é tirado do gráfico.

$$
i_{3}=\sqrt{i_{1} \cdot i_{2}}
$$

Foram obtidos $i_{3}=1,1 \mathrm{~mm} / \mathrm{min}$ e $t_{3}=39,0 \mathrm{~min}$.

Colocando-se na Equação 9 os valores de $i_{1}, i_{2}$ e $i_{3}$ obtidos da Equação Geral (Eq.-1), tem-se a Equação 12:

$$
\frac{A}{\left(t_{3}+c\right)^{b}}=\sqrt{\frac{A}{\left(t_{1}+c\right)^{b}} \cdot \frac{A}{\left(t_{2}+c\right)^{b}}}
$$
Equação 13: 


$$
c=\frac{t_{3}^{2}-t_{1} \cdot t_{2}}{t_{1}+t_{2}-2 t_{3}}
$$

O valor de $c$ obtido foi 28,3 .

\section{Verificação do valor de c e obtenção de um valor provisório para $\mathrm{b}$}

O próximo passo foi verificar se o valor de $c$ adotado era satisfatório. A verificação é feita traçando uma nova curva com os pontos $(t+c, i)$, o que equivale a deslocar horizontalmente para a direita todos os pontos da curva provisória. $\mathrm{O}$ valor $c$ obtido é considerado satisfatório quando os pontos deslocados configurarem aproximadamente uma linha reta (curva ajustada da Figura 2).

A inclinação da reta traçada fornece um valor provisório para o parâmetro $b$. No caso consideraram-se os pares de pontos $\left(t_{1}=100\right.$ min; $i_{1}=0,9$ $\mathrm{mm} / \mathrm{min})$ e $\left(t_{2}=34 \mathrm{~min} ; i_{2}=2,4 \mathrm{~mm} / \mathrm{min}\right)$ resultando no valor de $b$ dado pela Equação 14 .

$$
b=\frac{\log 2,4-\log 0,9}{\log 100-\log 34}=0,909
$$

Obtenção do parâmetro A=B.T $\mathrm{T}^{\mathrm{d}}$ do valor definitivo de $b$

Na determinação da constante $A$ e do valor definitivo de $b$, foi empregado o método dos mínimos quadrados, representado pelas equações 15 e 16 abaixo:

$$
\log A=\frac{\sum \log i \cdot \sum[\log (t+c)]^{2}-\sum \log (t+c) \cdot \sum[\log i \cdot \log (t+c]}{n \sum[\log (t+c)]^{2}-\left[\sum \log (t+c)\right]^{2}}
$$

$$
b=\frac{\sum \log (t+c) \cdot \sum \log i-n \cdot \sum[\log i-\log (t+c)]}{n-\sum[\log (t+c)]^{2}-\left[\sum \log (t+c)\right]^{2}}
$$

A forma geral da equação de chuva apresentada na Equação 3 passa a ter a configuração da
Equação 17, proposta por Wilken (1978), na qual a constante $A$ definida nas equações 8 e 9 para a ser representada por: $A=B . T^{d}$. Comparando-se a Equação 16 com a Equação 3, B substitui $\mathrm{K}, d$ substitui $m, c$ substitui $t_{0}$ e $b$ substitui $n$ nessa nova nomenclatura de variáveis.

$$
i=\frac{A}{(t+c)^{2}}=\frac{B \cdot T^{d}}{(t+c)^{b}}
$$

Onde

$i=$ intensidade de precipitação $\mathrm{em} \mathrm{mm} / \mathrm{h}$;

$T=$ período de retorno em anos

$t=$ duração da chuva em minutos

$B, c, b$ e $d$ = parâmetros particulares da equação para cada localidade

Na determinação do valor da constante $B$ e da constante $d$, foi tirada a média dos valores de $A$ referentes a cada período de retorno, isto é, para cada período de retorno foi tirado um valor médio de $A$. Empregou-se novamente o método dos mínimos quadrados na determinação das constantes $B$ e $d$ dado pelas equações 18 e 19 .

$$
\log b=\frac{\sum(\log T)^{2} \cdot \sum \log A-\sum(\log A \cdot \log T) \cdot \sum \log T}{n \cdot \sum(\log T)^{2}-\left(\sum \log T\right)^{2}}
$$

$$
d=\frac{n \cdot \sum(\log A \cdot \log T)-\sum \log T \cdot \sum \log A}{n \cdot \sum(\log T)^{2}-\left(\sum \log T\right)^{2}}
$$

Nesta fase de emprego do método dos mínimos quadrados, foi utilizado o programa computacional EQCHUD (Equações de Chuvas - Desenvolvimento) elaborado por Silva (1990). Finalmente, os parâmetros da nova equação de chuva para Fortaleza resultaram em: $c=28,31 ; b=0,904$; $B=2.345,29 ; d=0,173$, os quais se aplicam à Equação 17, resultando na Equação 20. 


\section{Resultados e discussões}

A Equação 20 representa a equação de chuvas intensas para Fortaleza desenvolvida com base em 30 anos de registros pluviográficos contínuos (1970 a 1999) segundo a metodologia apresentada.

$$
i=\frac{2345,29 \cdot T^{0,173}}{(t+28,31)^{0,904}}
$$

Onde

$i$ = intensidade média de chuva em $\mathrm{mm} / \mathrm{h}$ para a duração da chuva de t minutos

$T$ denota o período de retorno da precipitação em anos

Essa equação é apresentada graficamente na Figura 3

\section{Comparação entre as quatro equações}

As figuras 4 e 5 mostram os gráficos com as curvas I-D-F relativas aos períodos de retorno de 50 anos e 100 anos calculadas pelas três equações de chuvas existentes de Fortaleza e a equa- ção proposta, respectivamente.

Os gráficos das figuras 4 e 5 mostram que não há uma diferença entre as intensidades de precipitações calculadas pelas quatro equações para cada período de retorno respectivo, a partir da duração de 10 minutos. A equação mais antiga da AUMEF (CEARÁ,1978) corresponde à envoltória inferior das intensidades de precipitações para cada duração e período de retorno, enquanto que a equação de Rodrigues (2008) corresponde à envoltória superior destas intensidades. A equação proposta pelos autores praticamente adere à curva representativa da equação de Matos Neto e Fraga (1983) e situa-se entre as duas envoltórias, aderindo praticamente à curva da equação desenvolvida por Rodrigues a partir de uma duração de chuva de 60 minutos.

A maior diferença percentual entre as intensidades de precipitação calculadas pela equação proposta pelos autores e as outras equações ocorre para a duração de 5 minutos quando comparada com a equação de Rodrigues (2008) para um período de retorno de 100 anos (27\%). Esta diferença percentual se reduz até um mínimo de 2,5\% para durações de chuva superiores a 60 minutos.

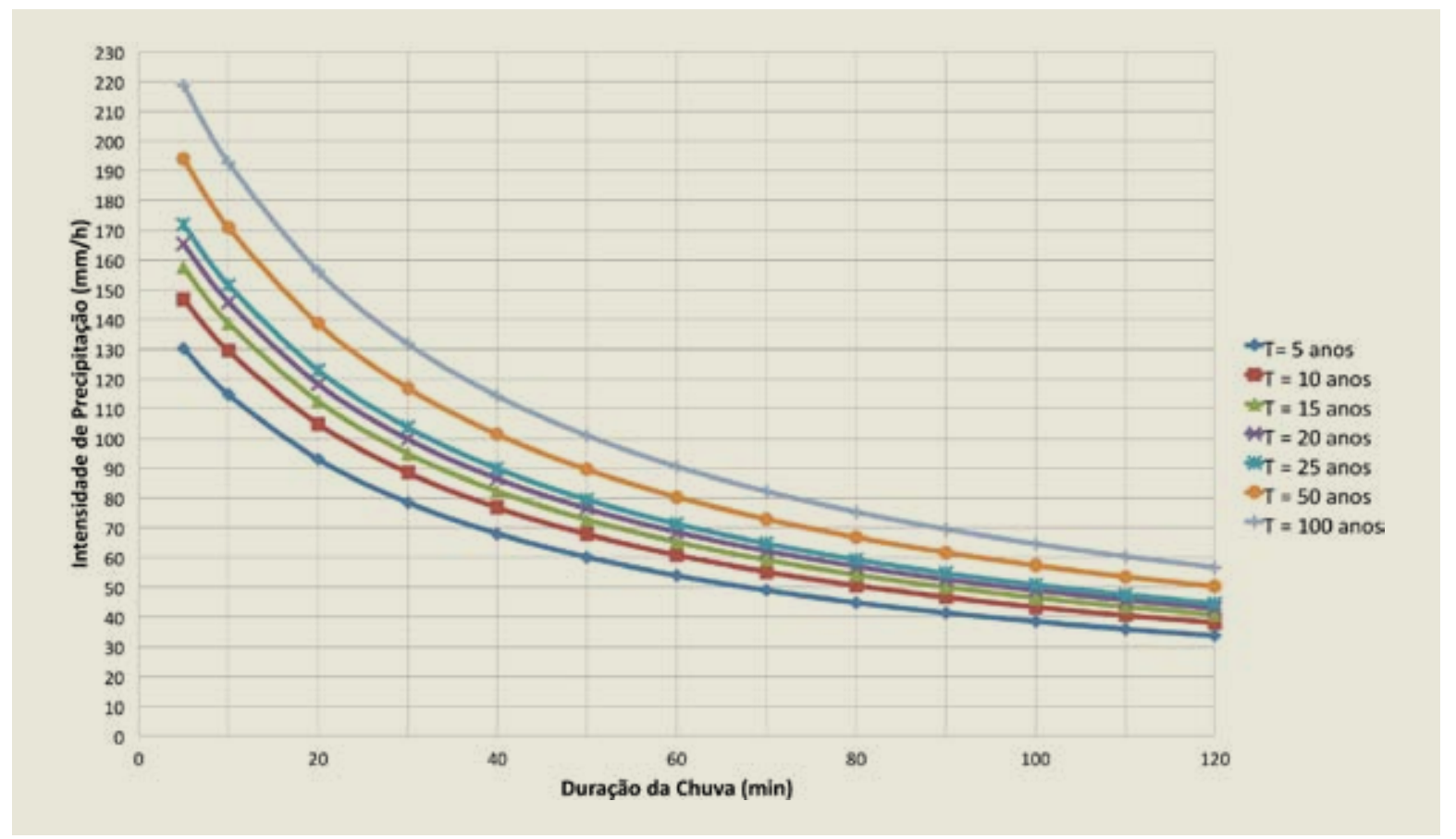

Figura 3 - Curva de intensidade-duração-frequência de chuvas proposta para a estação climatológica da UFC em Fortaleza- CE 


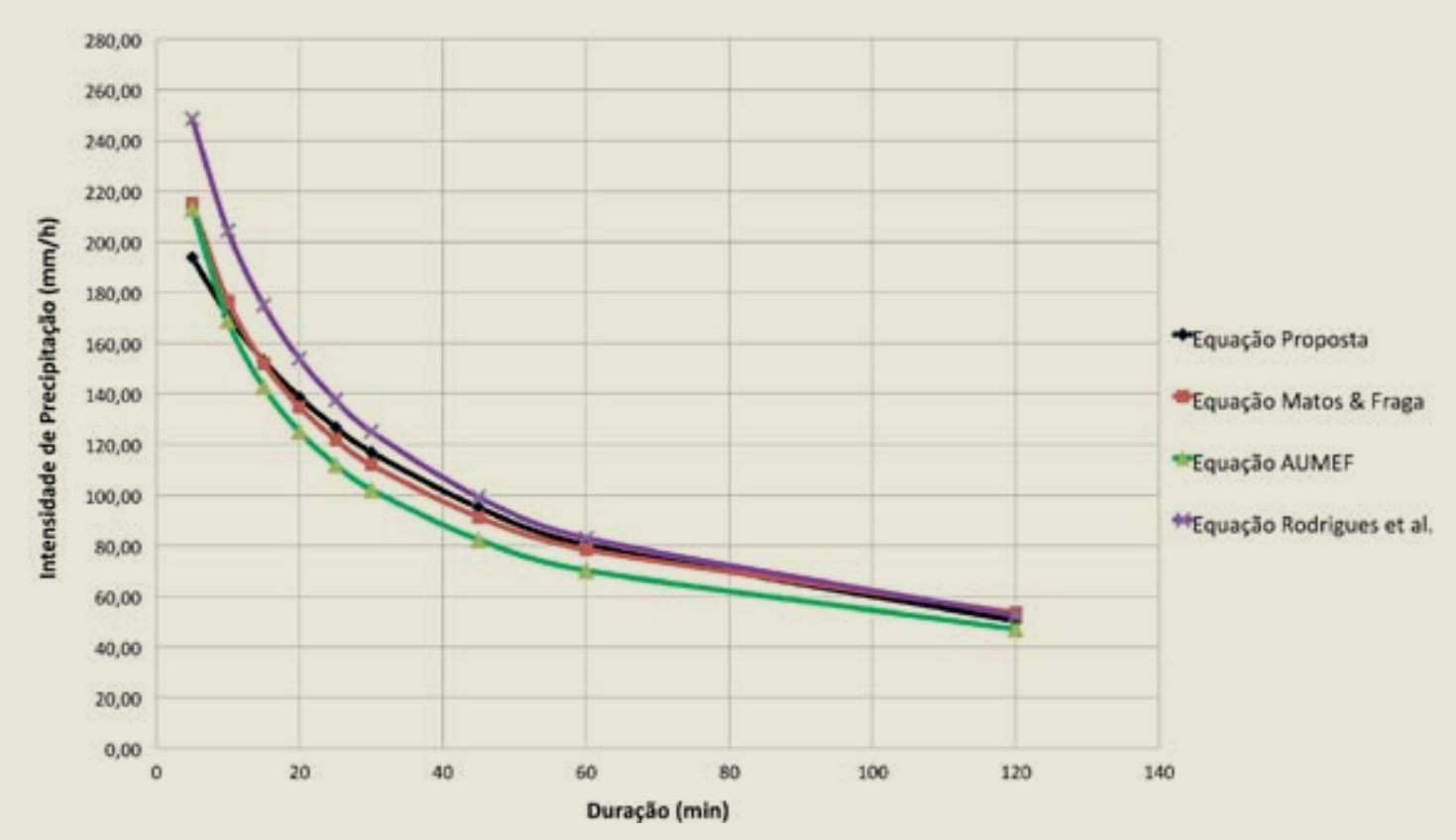

Figura 4- Curvas I-D-F das quatro equações de Fortaleza para o período de retorno de 50 anos.

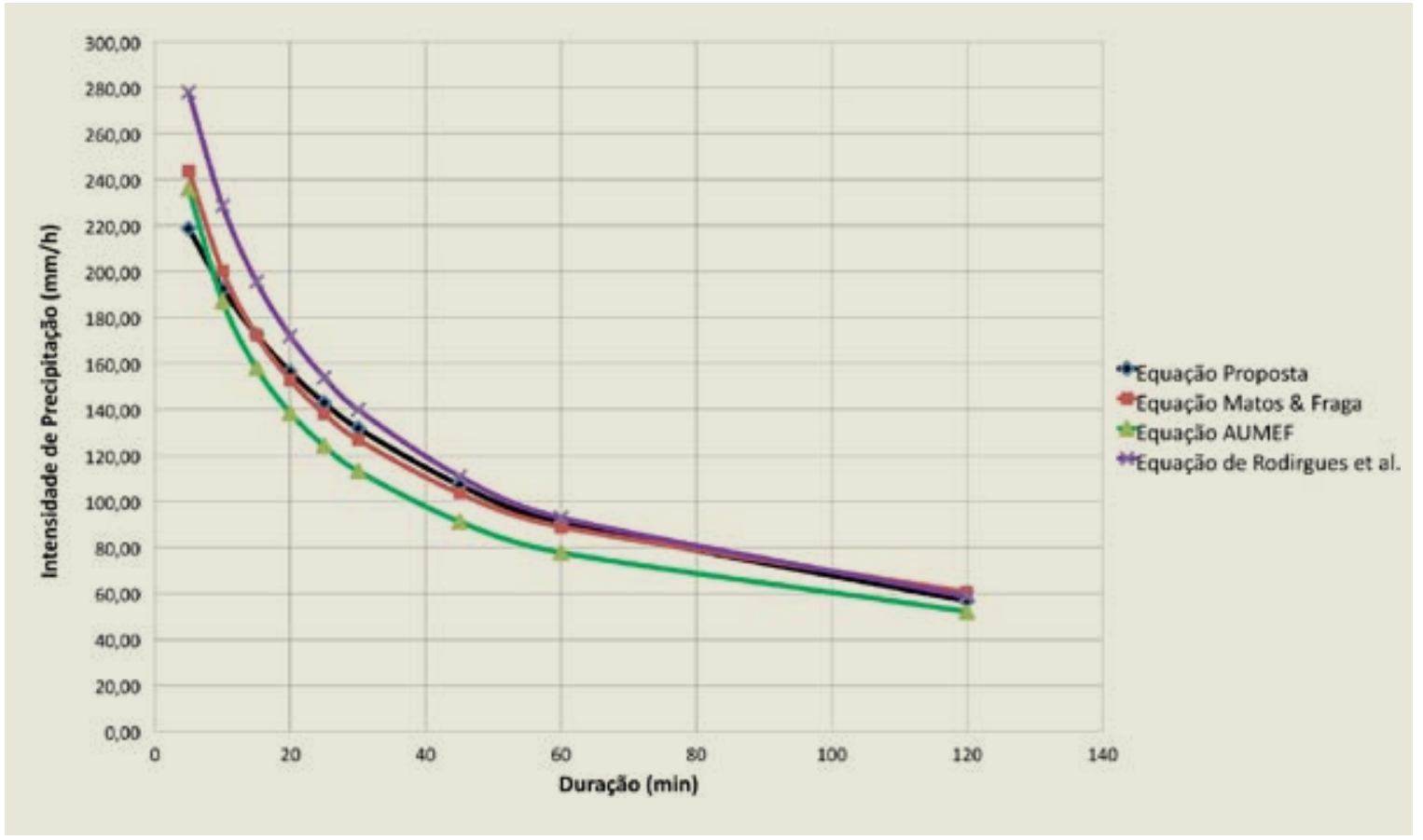

Figura 5- Curvas I-D-F das quatro equações de Fortaleza para o período de retorno de 100 anos. 


\section{Conclusões}

A partir da análise das alturas de chuvas diárias com durações sucessivas de 5 minutos foram desenvolvidas curvas que representam a relação entre intensidade, duração e frequência de chuvas intensas para a cidade de Fortaleza. Foi ajustada uma equação que representa estas curvas IDF. A equação obtida permite o cálculo da intensidade máxima de chuvas com durações entre 5 e 120 minutos e períodos de retorno de 5 a 100 anos.

A nova equação proposta apresenta resultados dentro da envoltória de intensidades de precipitação, calculadas para uma mesma duração e período de retorno, pelas outras três equações de chuva para Fortaleza, desenvolvidas em 1976, 1983 e 2008. No entanto, apresenta uma tendência de incremento nas intensidades de precipitação superior às calculadas com a equação da AUMEF (CEARÁ, 1978), que ainda é aceita como a equação oficial de chuvas pelos órgãos de infraestrutura de Fortaleza, para projetos de drenagem urbana.

O estudo comprovou que as quatro equações de chuva desenvolvidas para Fortaleza não apresentam resultados que diferem significativamente do ponto de vista estatístico e que todas são adequadas para emprego em projetos de obras de microdrenagem urbana em pequenas bacias de drenagem em Fortaleza e sua região metropolitana. $\mathrm{O}$ projeto de obras de maior envergadura como pontes e barragens que envolvam bacias de drenagem com áreas superiores a 2,5 km² deve considerar metodologias mais específicas que considerem a distribuição espacial dos postos pluviométricos e a extensão de suas séries históricas.

É importante salientar a necessidade de revisões futuras das equações de chuva, à medida que sejam incorporados novos dados de chuvas às séries históricas disponíveis, permitindo investigar uma possível tendência de elevação de eventos extremos na região. A existência de equação de chuvas atualizada para fins de auxílio aos estudos hidrológicos é de fundamental importância para evitar o subdimensionamento de projetos hidráulicos e de drenagem. A aparente tendência incremental nas intensidades de precipitação resultantes da ordem cronológica de desenvolvimento das quatro equações de chuva disponíveis para Fortaleza mostra a necessidade de se proceder periodicamente uma revisão destas equações conforme destaca Zuffo (2004).

\section{Referências}

BACK, A. J. Relações Entre Precipitações Intensas de Diferentes Durações Ocorridas no Município de Urussanga, SC.: Rev. Brás. Eng. Agríc. Ambient. Vol.13 no. 2 Campina Grande Mar. Apr. 2009.

BELL, F.C. Generalized rainfall-duration-frequency relationships. Journal of the Hydraulics Division, 95: 311-327, 1969.

BEVEN, K., LAMB, R., QUINN, P., ROMANOWICZ, R., FREER, J., TOPMODEL.

In: SINGH, V.P. (ed.). Computer Models of Watershed Hydrology. Water Resource

Publications, Colorado, p.627-668, 1995.

BRASIL, Instituto Brasileiro de Geografia e Estatística, IBGE Cidades, disponível em: http://www.ibge. gov.br/cidadesat/, acesso em março/2011.

CAMPOS, J.N.B. Lições em Modelos e Simulação Hidrológioca. Fortaleza, ASTEF, 2009. 166 p.

CEARÁ. Instituto de Pesquisa e Estratégia Econômica do Ceará. Estatísticas do Ceará. Disponível em: http://www.ipece.ce.gov.br. Acesso em março/2011.

CEARÁ. Secretaria do Planejamento. Autarquia da Região Metropolitana de Fortaleza. Plano Diretor de Drenagem na Região Metropolitana de Fortaleza. Fortaleza, 1978. 269 p.

CHOW, V. T. Statistical and probability analysis of hydrologic data: part I Frequency analysis In. CHOW, V. T. (ed) Handbook of applied hydrology. New York: MacGraw-Hill. Section 8, p. 1-42, 1964.

COSTA, A.R.; BRITO, V.F. Equações de Chuva Intensa para Goiás e Sul de Tocantins. In Simpósio Brasileiro de Recursos Hídricos, 13., 1999. Belo Horizonte.

COSTA, A.R.; RODRIGUES, A.A. Método as Isozonas: Desvios entre Resultados. In: Simpósio Brasileiro de Recursos Hídricos, 13. 1999,. Belo Horizonte.

CITYMAYORS. List of the 100 bigger cities in the world. Disponível em: http://www.citymayors.com. Acesso em junho/2010.

DAMÉ, R. C. F.; PEDROTTI, C. B. M.; CARDOSO, M. A.; SILVEIRA, C. P.; DUARTE, L. A.; MOREIRA, A. C. Comparação entre curvas intensidade-Duração-Frequência de ocorrência de precipitação obtidas a partir de dados pluviográficos com aquelas estimadas por técnicas de desagregação de chuva diária. 
Revista Brasileira de Agrociência, v.12, n.4, p.505509, 2006.

DNIT, Departamento Nacional de Infraestruturas de Transportes; Diretrizes Básicas para Elaboração de Estudos e Projetos Rodoviários - Escopo Básico/ Instruções de Serviço. Instituto de Pesquisas Rodoviárias. Publicação IPR-726. Brasília. 487p. 2006.

FENDRISCH, R., OBLADEN, N. L., AISSE, M. M., GARCIAS, C. M. Drenagem e controle da erosão urbana. 3. ED. São Paulo: Ibrasa. Curitiba: CHAMPAGNATI, 1991. $442 \mathrm{p}$.

GARCEZ; L. N. Hidrologia. São Paulo: Edgar Blucher ltda., 1974. 274p.

MARTINEZ JÚNIOR, F. Análise das Precipitações Intensas no Estado de São Paulo. In: Simpósio Brasileiro de Recursos Hídricos, 13., 1999 Belo Horizonte.

MATOS NETO, C. E. A.; FRAGA, N. S. Equação de Chuvas Intensas para a Cidade de Fortaleza. In: V Simpósio Brasileiro de Hidrologia e Recursos Hídricos, 1983, Blumenau-SC. Anais V Simpósio Brasileiro de Hidrologia e Recursos Hídricos, 1983.

LIMA, V.P.M. Variabilidade horo-sazonal da precipitação de Fortaleza-Ce em anos de contrastes. Dissertação de Mestrado. Departamento de Engenharia Hidráulica e Ambiental: Universidade Federal do Ceará. Fortaleza, Ce 2005.

MAGNI, N.L.; MERO,F. Precipitações intensas no Estado de São Paulo. Boletim no 4 DAEE/FCTH/ EPUSP, São Paulo, 1986.

MARTINEZ Jr, F.M. e MAGNI, N.L.G. Equações de Chuvas Intensas no Estado de São Paulo. Relatório Técnico do Convênio entre Departamento de Águas e Energia Elétrica de São Paulo e a Escola Politécnica da Universidade de São Paulo (EPUSP). São Paulo, SP, 1999. 125p.

NERILO, N.; MEDEIRO, P.A.; CORDEIRO, A.; Chuvas intensas no Estado de Santa Catarina. Edifurb. Florianópolis. 2002. 156p.

OLIVEIRA, C.P.M; PORTO, R.L.; ZAHED FILHO,K; ROBERTO,A.N., ABC6, um sistema de suporte a decisões para análise de cheias em bacias complexas. XIII Simpósio Brasileiro de Recursos Hídricos, Belo Horizonte, 1999.

OLIVEIRA. L.F.C. et al. Estimativa das equações de chuvas intensas para algumas localidades no Estado de Goiás pelo método da desagregação das chuvas. Pesquisa Agropecuária Tropical, 30 (1): 23-27, jan./ jun. 2000.

PFAFSTETTER, O. Chuvas Intensas no Brasil. Relação entre Precipitação, Duração e Frequência de Chuvas Registradas com Pluviográfos em 98 Postos Meteorológicos. 2. ed. Rio de Janeiro: Departamento Nacional de Obras de Saneamento, 1982. 426 p.

PINTO, F.A., et al. Equações de chuvas intensas para algumas localidades do Estado de Minas Gerais. Engenharia Agrícola. 16(1): 91-104. 1999.

PONCE, V.M. Engineering Hydrology: Principles and Practices. New York.:Prentice Hall 1989. 640 p.

RODRIGUES, J.O.; ANDRADE, E.M.; OLIVEIRA, T.S.; LOBATO, A.O.; Equações de Intensidade-Duração-Frequência de Chuvas para as Localidades de Fortaleza e Pentecoste, Ceará. Scientia Agrária. Curitiba. v.9, n.4, p.511-519. 2008.

SILVA, D.D.; VALVERDE, A.E.L.; PRUSKI, F.F.; GONÇALVES, R.A.B. Estimativa e Espacialização dos Parâmetros da Equação de intensidade-duração-freqüência da Precipitação para o Estado de São Paulo. Engenharia na Agricultura, Viçosa, v.7, n.2, p.70-87, 1999a.

SILVA, F.O.E. Notas de Aula de Hidrologia Aplicada. Fortaleza: Universidade de Fortaleza, 2007.

SILVA, F.O.E. Programa EQCHUD - Desenvolvimento de Equações de Chuva. Mestrado em Recursos Hídricos, Universidade Federal do Ceará, Fortaleza, 1990.

SOUZA, F. A. S.; SILVA, V. P. R. Gestão e controle ambiental. Revista Brasileira de Engenharia Agrícola e Ambiental, v.2, n.2, p.319-323, 1998.

TABORGA, J.T., Práticas Hidrológicas. Transcon. Rio de Janeiro. 1974

VILLELA, S. M; MATTOS, A. Hidrologia aplicada. São Paulo - Brasil: McGraw-Hill, 1975.

WILKEN, P. S. Engenharia de Drenagem Superficial. São Paulo: CETESB, 477p. 1978.

XAVIER, L.N.R. Análise da Incerteza Causada pela Representação da Precipitação no Modelo Topmodel. Dissertação de Mestrado em Ciências em Engenharia Civil. COPPE/Universidade Federal do Rio de Janeiro. Rio de Janeiro. 2002. 124p.

ZUFFO, A. C. EQUAÇÕES DE CHUVAS SÃO ETERNAS? In XXI Congresso Latino Americano de Hidráulica, São Pedro - SP. 2004. 